\title{
Management of infusion reactions associated with cetuximab treatment: A case report
}

\author{
NAOHIRO OHSHITA ${ }^{1}$, YURI ICHIMARU ${ }^{1}$, SHOKO GAMOH $^{2}$, KANAME TSUI $^{3}$, \\ NAOTAKA KISHIMOTO ${ }^{1}$, YASUO M. TSUTSUMI ${ }^{4}$ and YOSHIHIRO MOMOTA ${ }^{1}$ \\ Departments of ${ }^{1}$ Anesthesiology and ${ }^{2}$ Oral Radiology; ${ }^{3}$ First Department of Oral and Maxillofacial Surgery, \\ Osaka Dental University, Osaka 540-0008; ${ }^{4}$ Department of Anesthesiology, Faculty of Medicine, \\ Tokushima University, Tokushima 770-8503, Japan
}

Received September 21, 2016; Accepted March 6, 2017

DOI: $10.3892 /$ mco.2017.1242

\begin{abstract}
Cetuximab is a drug targeting the epidermal growth factor receptor, which is indicated for the treatment of unresectable advanced or recurrent head and neck or colorectal cancer. Cetuximab also enhances the cytotoxic effects of radiation in squamous cell carcinoma. The severity of infusion reactions (IR) is categorized from grade 1 to 5; grades 3 and 4 are associated with life-threatening reactions (anaphylaxis), whereas grade 5 indicates death. The incidence of grade 3-4 IR with premedication is reported to be $1.1 \%$. We herein describe a case of a 77-year-old man who developed IR during intravenous administration of cetuximab. The patient developed grade 3-4 anaphylaxis with pruritus, rash and urticaria, followed by hypotension and bradycardia. The timely diagnosis and treatment with intravenous infusion of a vasopressor drug and Ringer's acetate solution proved to be effective. The case presented herein demonstrated an unfeatured aspect of cetuximab-related IR as dermatological reactions over the entire body followed by circulatory collapse.
\end{abstract}

\section{Introduction}

In Japan, cetuximab has been established as a non-surgical treatment option for head and neck cancer in combination with radiotherapy since December, 2012. Cetuximab is expected to be effective in the long-term management of patients with recurrent or metastatic head and neck cancer; however, adverse reactions such as sudden death, arrhythmia, degeneration of interstitial pneumonia and hypomagnesaemia are commonly observed (1). Therefore, it is crucial to identify and address those symptoms in the early stages. We herein present a case

Correspondence to: Dr Shoko Gamoh, Department of Oral Radiology, Osaka Dental University, 1-5-17 Otemae, Chuo-ku, Osaka 540-0008, Japan

E-mail: gamo-s@cc.osaka-dent.ac.jp; shoko1105@gmail.com

Key words: cetuximab, epidermal growth factor receptor, galactose- $\alpha-1,3$-galactose, head and neck cancer, infusion reactions of infusion reactions (IR) induced by cetuximab (Erbitux ${ }^{\circledR}$ ) from an anesthesiologist's viewpoint. The patient was treated for the IR by administration of ephedrine alone, which is an uncommon method for treating IR (2). The difficulties of diagnosing and managing IR are also discussed, with a brief review of the literature.

\section{Case report}

The patient was a 77-year-old man $(160 \mathrm{~cm}$ in height, $57 \mathrm{~kg}$ in weight, body surface area $1.591 \mathrm{~m}^{2}$ ). The patient had previously undergone tracheotomy, resection of the left part of the tongue, left radical neck dissection and reconstructive surgery with pectoralis major myocutaneous flaps for the treatment of left-sided tongue cancer, and had received 3 cycles of chemotherapy with cisplatin and TS-1. A lymph node metastasis was found in the right neck within 6 months after the surgery, and intravenous infusion of cetuximab in the outpatient clinic was planned. The patient had been medicated with voglibose, glimepiride and clopidogrel for diabetes (HbA1c 6.0) and atherosclerosis obliterans of the lower extremities.

Upon arrival at the outpatient clinic, the patient's blood pressure (BP) was $140 / 76 \mathrm{mmHg}$, the heart rate (HR) was $60 \mathrm{bpm}$ and the blood oxygen saturation $\left(\mathrm{SpO}_{2}\right)$ was $96 \%$. Peripheral venous access was obtained with an $22 \mathrm{G}$ indwelling needle on the forearm, and D-chlorpheniramine maleate $5 \mathrm{mg}$, dexamethasone sodium phosphate $13.2 \mathrm{mg}$ and azasetron hydrochloride $10 \mathrm{mg}$ were infused intravenously over $1 \mathrm{~h}$. The infusion of cetuximab $600 \mathrm{mg}$, which represents the loading dose of $400 \mathrm{mg} / \mathrm{m}^{2}$, was then initiated at a rate of $5 \mathrm{mg} / \mathrm{min}$. Ten minutes after starting the infusion, facial flushing developed and the patient complained of pruritus on the abdomen. The vital signs were stable (BP, 124/68 $\mathrm{mmHg}$; HR, $75 \mathrm{bpm}$; and $\mathrm{SpO}_{2}, 96 \%$ ). According to the instruction manual, cetuximab infusion was continued at a reduced rate of $2 \mathrm{mg} / \mathrm{min}$. A few minutes later, diaphoresis developed and the pruritus worsened, with severe erythema and raised rash on the trunk and extremities (Fig. 1). The BP had decreased to $62 / 36 \mathrm{mmHg}$ and the HR to $48 \mathrm{bpm}$; therefore, an anaphylactoid reaction was suspected. Cetuximab infusion was discontinued immediately, and the infusion solution was replaced with Ringer's acetate solution. A total of $16 \mathrm{mg}$ methylephedrine was administered. 
The patient was sufficiently lucid to breath normally without nausea. Although no decrease in $\mathrm{SpO}_{2}$ was noted, he was administered oxygen through a Polymask at $61 / \mathrm{min}$ to prevent hypoxia. After methylephedrine hydrochloride administration and rapid fluid infusion of $500 \mathrm{ml}$, the patient recovered from the circulatory collapse with a BP of $119 / 63 \mathrm{mmHg}$ and a HR of $63 \mathrm{bpm}$. The vital signs were stabilized (BP, 122-148/66-78 mmHg; HR, 62-76 bpm; $\mathrm{SpO}_{2}, 99 \%$ in room air), and the patient was hospitalized at the Osaka Dental University Hospital (Osaka, Japan), whereas a dermatologist at an affiliated hospital was consulted. In addition to prescription of olopatadine hydrochloride and betamethasone butyrate propionate, the patient received an intravenous infusion of $250 \mathrm{mg}$ methylprednisolone sodium succinate. During hospitalization, mild erythema persisted on the back, foreneck and chest, but the raised rash had disappeared before discharge on the following day.

\section{Discussion}

Our case presented two interesting clinical points: Methylephedrine may be useful for the treatment of anaphylactoid reactions when administered alone, and the patient's living environment is significantly associated with the incidence of IR.

This case provided us with a valuable opportunity to determine whether ephedrine hydrochloride administration was effective to reverse the grade 3-4 IR, as epinephrine, rather than ephedrine, has been commonly used for the treatment of IR. Ephedrine is a vasoconstrictor generally used to restore the BP to normal levels. The incidence of IR has been reported to be $15 \%$ for all grades of IR, and $3 \%$ for IR of grade $\geq 3$ (3). Pre-treatment with antihistamines and corticosteroids is often performed as a standard method to reduce the risk of IR associated with cetuximab treatment; in colorectal cancer patients, the incidence of grade 3-4 IR was found to be lower, namely $1.1 \%$ (4). However, symptoms associated with IR may occur despite prophylaxis, as this patient developed a reaction to cetuximab despite premedication. As regards the development of IR, previous reports suggested that IR developed within $1 \mathrm{~h}$ after starting the cetuximab infusion, particularly within the first $15 \mathrm{~min}$ (4). The patient in the present case experienced the development of IR within $20 \mathrm{~min}$ after treatment initiation. The treatment of IR depends on the severity, and grade $\geq 3$ symptoms require treatment similar to that for anaphylaxis $(1,4)$. In this case, the severity of the symptoms increased even after reducing the infusion rate of cetuximab according to the instruction manual. It was crucial that the surgeon as well as the dental anesthesiologist carefully observed the patient in order to assess the situation in the early stage. The key charateristic was circulatory collapse following dermatological reactions. Respiratory events, such as airway obstruction and edema, were not evident. Ephedrine, which was ready for emergency use, was initially administered, and the BP and HR gradually increased following additional dosage of ephedrine with rapid infusion of acetate Ringer's solution. The vital signs were stabilized by administering a total dose of $16 \mathrm{mg}$ ephedrine. D-chlorpheniramine and dexamethasone as prophylaxis may be helpful in restoring BP and $\mathrm{HR}$, enhancing the vasopressor effect of ephedrine. In case the

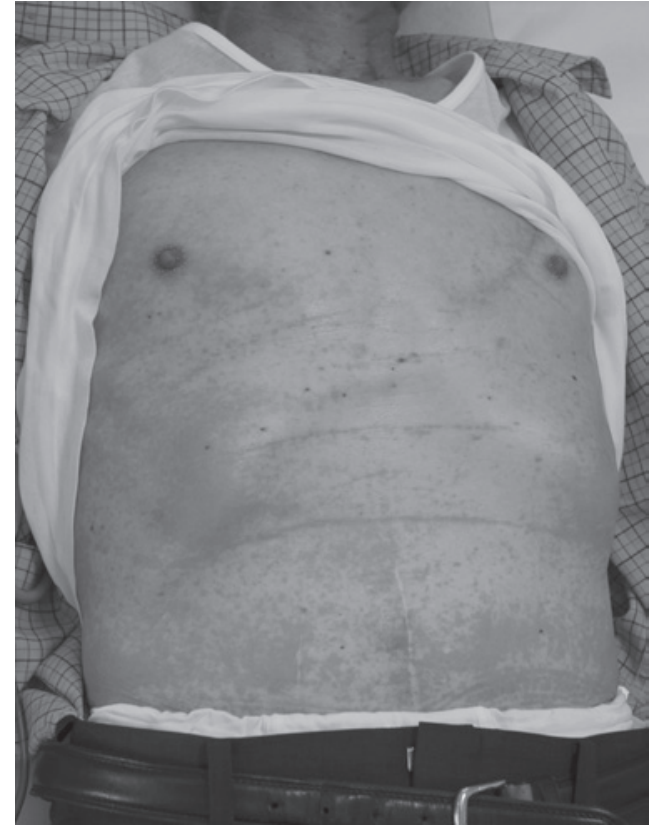

Figure 1. Pruritus, rash and urticaria were observed on the patient's skin during cetuximab infusion.

BP and HR were unresponsive to ephedrine, a different vasopressor would have to be considered; an $\alpha$ - and $\beta$-stimulator, such as epinephrine, is considered to be a safe method for controlling respiratory events.

This case also suggested a correlation between the patient's living environment and high incidence of IR. Certain factors in the living environment may act as antigen towards cetuximab, eventually increasing the risk of IR by sensitizing the patient. For example, reports from the United States have described that the prevalence of anti-cetuximab $\operatorname{IgE}$ antibodies varies in different geographical areas, and hypothesized that the incidence of allergic reactions to cetuximab may be related to the incidence of allergic reactions to red meat and Rocky Mountain spotted fever, and the habit distribution of ticks carrying Rickettsia rickettsii, the bacterium causing Rocky Mountan spotted fever (5). It has been reported that IgE antibodies against galactose- $\alpha-1,3$-galactose ( $\alpha$-gal) elicit anaphylaxis in patients receiving cetuximab $(5,6)$. The fact that patients who develop IR during the first infusion of cetuximab were found to have $\operatorname{IgE}$ antibodies specific to cetuximab, indicates that they had been previously exposed to sensitizers $(5,6) ; \alpha$-gal is present in cows and pigs, and is believed to cause late-onset urticaria and anaphylaxis that develop in 3-6 h after eating beef or pork. It is also believed that the level of beef-specific IgE antibodies is correlated with the level of cetuximab-specific $\operatorname{IgE}$ antibodies $(5,7,8)$. The level of $\alpha$-gal-specific IgE antibodies is also correlated with the level of IgE antibodies specific to the tick Amblyomma americanum, a vector of Rocky Mountain spotted fever that is commonly found in the southwestern to eastern part of the United States (4). These findings indicate that antibodies in patients with a meat allergy, or those with a history of tick bite, may cross-react against cetuximab (5-8). In Japan, mites are drawing attention as a cause of a syndrome combining severe fever with thrombocytopenia in recent years. However, a report 
from the Shimane Prefecture suggests that there are overlaps in areas where spotted fever is prevalent and those where several patients with beef allergy reside (9). That study also reported that $75 \%$ of patients with beef allergy are also allergic to flounder eggs, which indicates that anti-beef antibodies may cross-react with flounder egg antigens (9). As a number of these patients have lived with dogs and have $\operatorname{IgE}$ antibodies specific to dog's skin, the authors of that study concluded that mites, which bite dogs as well as humans, may induce beef allergy (9). Altogether, reports from the United States as well as Japan suggest that several factors in the living environment are associated with the incidence of IR to cetuximab.

Therefore, physicians must question the patients on past illnesses prior to administering cetuximab. Unfortunately, the patient in the present case received cetuximab infusion without a detailed consultation on the risk factors for IR. A consultation after the onset of IR revealed that he was from Izumo (Shimane, Japan). In addition, male gender and smoking are factors known to increase the risk of IR (10), indicating that this patient was at a higher risk of developing IR.

In conclusion, this case illustrated the usefulness of ephedrine in managing IR symptoms during the first administration of cetuximab for the treatment of a metastatic lymph node in the neck. The present case has also highlighted the importance of interviewing the patient on past and present places of residence, history of allergic reactions to beef and/or flounder eggs, history of tick bites and history of living with dogs prior to initiating cetuximab therapy.

Informed consent was obtained from the patient regarding the publication of the details of this case and associated images.

\section{References}

1. Lenz HJ: Management and preparedness for infusion and hypersensitivity reactions. Oncologist 12: 601-609, 2007.

2. Enokibori M, Kuge M and Mori K: Anaphylactoid reaction to maltose 5\% solution during spinal anaesthesia. Can J Anaesth 45: 52-55, 1998.

3. Bonner JA, Harari PM, Giralt J, Azarnia N, Shin DM, Cohen RB, Jones CU, Sur R, Raben D, Jassem J, et al: Radiotherapy plus cetuximab for squamous-cell carcinoma of the head and neck. $N$ Engl J Med 354: 567-578, 2006.

4. Yamaguchi K, Watanabe T, Satoh T, Ishiguro M, Izawa M, Inoshiri S, Sugihara K and Sakata Y: Severe infusion reactions to cetuximab occur within $1 \mathrm{~h}$ in patients with metastatic colorectal cancer: Results of a nationwide, multicenter, prospective registry study of 2126 patients in Japan. Jpn J Clin Oncol 44: 541-546, 2014.

5. Commins SP, James HR, Kelly LA, Pochan SL, Workman LJ, Perzanowski MS, Kocan KM, Fahy JV, Nganga LW, Ronmark E, et al: The relevance of tick bites to the production of $\operatorname{IgE}$ antibodies to the mammalian oligosaccharide galactosea-1,3-galactose. J Allergy Clin Immunol 127: 1286-93.e6, 2011.

6. Chung CH, Mirakhur B, Chan E, Le QT, Berlin J, Morse M, Murphy BA, Satinover SM, Hosen J, Mauro D, et al: Cetuximab-induced anaphylaxis and IgE specific for galactosealpha-1,3-galactose. N Engl J Med 358: 1109-1117, 2008.

7. Commins SP and Platts-Mills TA: Anaphylaxis syndromes related to a new mammalian cross-reactive carbohydrate determinant. J Allergy Clin Immunol 124: 652-657, 2009.

8. Commins SP, Satinover SM, Hosen J, Mozena J, Borish L Lewis BD, Woodfolk JA and Platts-Mills TA: Delayed anaphylaxis, angioedema, or urticaria after consumption of red meat in patients with IgE antibodies specific for galactose-alpha1,3-galactose. J Allergy Clin Immunol 123: 426-433, 2009.

9. Chinuki Y, Takahashi H and Morita E: Clinical and biochemical evaluation of twenty patients with red meat allergies. Jpn J Dermatol 123: 1807-1814, 2013.

10. Hopps S, Medina P, Pant S, Webb R, Moorman M and Borders E: Cetuximab hypersensitivity infusion reactions: Incidence and risk factors. J Oncol Pharm Pract 19: 222-227, 2013. 